\title{
BODY COMPOSITION AND POSTURAL INSTABILITY IN PEOPLE WITH IDIOPATHIC PARKINSON'S DISEASE
}

\author{
A.S. Diab, L.A. Hale, M.A. Skinner, G. Hammond-Tooke, A.L. Ward, D.L. Waters
}

\begin{abstract}
Objectives: Idopathic Parkinson's disease (PD) is the second most common neurodegenerative disorder. Our objective was to investigate the relationship between body composition and postural instability in people with PD, and age- and sexmatched controls. Design: Cross-sectional study among PD sufferers and age- and sex-matched controls. Setting: University of Otago's Balance Clinic, School of Physiotherapy. Participants: Forty-seven people with PD and 58 age- and sex-matched controls. Measurements: Postural stability was assessed with the Sensory Organization Test, Motor Control Test, Timed Up and Go Test, and Step Test. Body composition was measured by dual energy x-ray absorptiometry (DXA). Movement Disorders Society-Unified Parkinson's Disease Rating Scale was applied to assess PD severity. Results: Mean group differences between PD and controls for the equilibrium composite score, Timed Up and Go Tests, and Step Test were statistically significant ( $<<0.05)$; strategy and latency composite scores and body composition variables were not ( $\mathrm{p}>0.05$ ). Three PD participants were sarcopenic; 15 PD and 24 controls were obese. In PD participants, total body lean mass and age predicted latency composite scores. Disease, age, and leg fat mass predicted the Timed Up and Go Test results $(p<0.05)$. Sex and disease predicted the equilibrium composite $s c o r e(p<0.01)$. Conclusion: The prevalence of obesity was high and sarcopenia low in the PD group, which is a novel finding. Not surprisingly, participants with PD had reduced postural stability compared to controls. Disease status, age and sex were influential factors in the weak relationships found between postural stability and body composition. These findings may have clinical relevance for the treatment of the physical symptoms of those suffering from PD.
\end{abstract}

Key words: Body composition, postural instability, Parkinson's disease.

\section{Introduction}

Idiopathic Parkinson's disease (PD) is a common neurodegenerative disorder, which presents with a variety of motor and non-motor manifestations (1). Postural instability is considered a cardinal sign of PD, impacting independence and increasing falls risk (2). Postural instability typically presents late in PD, (3) although mechanisms for this timing are not fully understood. A number of factors have been suggested which include deficits in anticipatory and reactive responses to perturbations (4), visual and vestibular systems (5), sensory-motor integration (6), muscle tone (7), cognition (8), and muscle power (9). A recent review identified six primary factors contributing to postural instability in PD, dysfunction in sensory reorganization, bradykinesia, abnormal postural response patterns, L-dopa induced dyskinesia, hypotension, and cognitive impairment (10).

Centre for Health, Activity, and Rehabilitation Research, School of Physiotherapy, University of Otago, Dunedin, New Zealand.

Corresponding Author: Professor Leigh A. Hale, Centre for Health, Activity, and Rehabilitation Research, School of Physiotherapy, University of Otago, PO Box 56, Dunedin 9054, New Zealand, Tel:+64 3479 5425, Email: leigh.hale@otago.ac.nz

Postural instability is also prevalent in older adults without PD, resulting in increased risk of falling (11), and the associated factors are numerous (12). One possible factor identified in older adults is abnormal body composition phenotype, which is associated with losses in lean body mass and bone mineral density, and increases in fat mass and a sarcopenic-obese phenotype $(13,14)$. The association between reduced physical function and the sarcopenic obese phenotype has been widely reported $(15,16)$.

Body composition in PD has been investigated (17-20), especially in those with advanced stages of the disease (18), and it has been reported that people with PD are disproportionately sarcopenic (21). To our knowledge, no research has focused on the association between body composition phenotype and postural instability in PD. The aim of this study was to investigate this relationship.

\section{Methods}

\section{Study design}

A cross-sectional case-matched study compared people with PD to an age- and sex-matched control group. The 
Lower South Regional Ethics Committee, New Zealand approved the study.

\section{Participant recruitment}

Participants with PD were recruited through the Dunedin Hospital Neurology Department, the Parkinson Society, and via community advertising. Control participants were recruited via community advertising. Volunteers were screened for eligibility by telephone using a standardised checklist. Those meeting eligibility requirements were sent a study information sheet and assigned an appointment time, at which time eligibility and consent was confirmed. The diagnosis of PD was confirmed by the study neurologist. Inclusion criteria were age over 40 years, and the ability to perform the measurement tests independently with or without assistive mobility devices. Participants with other types of Parkinsonism, the inability to undergo the measurement tests safely due to cognitive or physical disability, or significant co-morbidity were excluded. Inclusion criteria for controls were age and sex-matched to the participants with PD, with no known neurological disorder, and self-reported to be sedentary.

\section{Study procedures}

All participants attended two appointments. Degrees of PD severity were assessed with the Movement Disorders Society-Unified Parkinson's Disease Rating Scale, and disease stage was defined by Hoehn and Yahr (22).

Postural instability was tested using both computerised posturography and clinical measures. Computerised posturography included the Sensory Organization Test (SOT) and the Motor Control Test (MCT) performed on the NeuroCom ${ }^{\circledR}$ Smart EquiTest ${ }^{\circledR}$ version 8.4.0. The clinical measures were the Timed Up and Go Test used without (TUG B) and with (TUG H) a concomitant cognitive task, which was naming the days of the week backwards, and the Step Test. The retest and inter-rate reliability of the TUG among people with PD has been found to be high during both L-dopa "off" and "on" states. The Step Test is a simple, reliable test commonly used for investigating dynamic stability and postural responses in older adults (23) and reliability has been reported to be good for those with PD and comparison groups (24).

Dual-energy X-ray Absorptiometry (DXA; Lunar DPX-L scanner GE LUNAR Corp; Madison, WI) was used to measure whole body and regional body composition. Prior to testing the scanner was calibrated per manufacturer protocol. Height and weight were measured using a standardized protocol. The appendicular skeletal muscle (ASM) was calculated by summing lean mass in the arms and legs. Phenotypes were determined by validated criteria. The sarcopenic phenotype was defined as ASM Index (ASM/height2) of $<7.26$ and $<5.4$ for males and females, respectively, and obese as an ASM Index of $\geq 27 \%$ and $\geq 38 \%$ for males and females, respectively (15).

\section{Data Analysis}

Data were entered into the SPSS statistics computer programme. Data from the postural stability tests and body composition were described in terms of mean and $95 \%$ CIs. The Student's T-test was used to compare the mean of the study and control groups for each test variable. Pearson's correlation coefficients (r) tests were used to test the strength and direction of association between the postural stability and body composition variables for the stepwise regression analysis. The selection criterion for choosing variables for regression was based on the results of the correlation analysis and informed by variables used in previously published research (25). Eight predictor variables (sex, age, disease, total body lean, total body legs lean, total body fat, percent body fat, and total body legs fat) used in the stepwise regression model.

\section{Results}

Table 1 shows participant characteristics from 47 participants with PD (male 57\%), and 58 control participants (male 34\%). Twenty-seven participants were in a moderate state (stage II) of PD, seven were in an advanced state (stage III-IV), and thirteen were in a mild state (stage I). Forty-one PD participants were on anti-Parkinsonian medications. No participants with PD had received deep-brain stimulation prior to the study. Dyskinesia was reported in those in stage II, and one participant in stage III. Dyskinesia was slight to mild and was self-reported not to impact on activities of daily living. Participants with PD who were on medication were categorized as in a state of "on" drug therapy.

Table 2 shows the mean and 95\% confidence intervals for the postural stability tests and the body composition variables. To maintain balance both groups were primarily using the ankle strategy $(79 \%)$ and without a delay, as the differences were non-significant for the strategy composite and latency composite scores. The mean Body Mass Index (BMI) was not significantly different between the PD and control groups.

There were no significant differences in the body composition variables. Males in the PD group had slightly less total fat mass than control males. The mean total fat mass in the PD female group was higher than the control females; however, these differences were not significant (Table 2). The number of females with obesity in both the PD and the control group were nearly double that of the males $(9 \%, 26 \%$, respectively). 
Table 1

Descriptive characteristics of the PD and Control groups

\begin{tabular}{|c|c|c|c|c|c|c|}
\hline \multirow{2}{*}{$\begin{array}{l}\text { Demographic parameters } \\
\text { Mean (SD or \%) }\end{array}$} & \multicolumn{3}{|c|}{ PD group $(n=47)$} & \multicolumn{3}{|c|}{ Control group $(n=58)$} \\
\hline & Male $n=27$ & Female $\mathrm{n}=\mathbf{2 0}$ & Total $n=47$ & Male $n=20$ & Female $n=38$ & Total $n=58$ \\
\hline Age years (SD) & $68.2(9.8)$ & $69.4(8.2)$ & $68.7(9.1)$ & $66.7(11.6)$ & $68.8(8.8)$ & $68.1(9.8)$ \\
\hline Height cm (SD) & $172.2(5.2)$ & $159.4(6.5)$ & $166.8(8.6)$ & $173(7.8)$ & $163(7.8)$ & $166.4(9.1)$ \\
\hline Weight kg (SD) & $77.3(12.8)$ & $72.1(15.4)$ & $75.1(14.1)$ & $77.3(11.8)$ & $69.6(14.5)$ & $72.3(14)$ \\
\hline Body mass index kg/m2 (SD) & $26.1(4.3)$ & $28.3(5.7)$ & $27(5)$ & $25.8(3.18)$ & $26.1(4.9)$ & $26.0(4.5)$ \\
\hline Disease duration (years) & 4.90 & 6.20 & $5.45(4.7)$ & & & \\
\hline \multirow[t]{2}{*}{ *MDS-UPDRS score } & 44.7 & 48.8 & $46.46(17.7)$ & & & \\
\hline & $n$ & $n$ & $n(\%)$ & & & \\
\hline \multicolumn{7}{|l|}{${ }^{* *}$ Disease staging } \\
\hline Stage I & 7 & 6 & $13(27.65)$ & & & \\
\hline Stage II & 17 & 10 & $27(57.44)$ & & & \\
\hline Stage III & 2 & 3 & $5(10.63)$ & & & \\
\hline Stage IV & 1 & 1 & $2(4.25 \%)$ & & & \\
\hline †»On" / “Off» state (Yes) & 9 & 7 & $16(34 \%)$ & & & \\
\hline \multicolumn{7}{|l|}{ Side affected first ( $n$ ) } \\
\hline Right side & 12 & 10 & $22(47 \%)$ & & & \\
\hline Left side & 12 & 10 & $22(47 \%)$ & & & \\
\hline Unsure & 2 & 1 & $3(6 \%)$ & & & \\
\hline Total number & 27 & 20 & 47 & 20 & 38 & 58 \\
\hline
\end{tabular}

*MDS-UPDRS: Movement Disorders Society -Unified Parkinson's Disease Rating Scale results; **Hoehn and Yahr; † «On” / "Off»: state of medical therapy

The three participants with PD in the sarcopenic phenotype were of relatively advanced age ( $\geq 75.6$ years) but varied in disease stage and severity of symptoms. Neither group presented as the sarcopenic obese phenotype. In fact, most presented as obese. There was a small positive relationship between total leg fat mass and the TUG B and TUG H scores. Disease, sex, and age were each found to be predictive of body composition in relation to postural stability. Disease predicted the equilibrium composite score, the TUG B and the TUG $\mathrm{H}$ scores, and age predicted the latency composite score and the TUG B and TUG $\mathrm{H}$.

Of the many variables considered for the prediction equation, only a small subset of variables was selected to obtain good predictive results to fit the model. Results revealed a very low to moderate level of multicollinearity between the predictors. The equilibrium composite variable was not significantly correlated in either the PD or the control groups, but was deemed necessary to keep in the regression model, as it is widely used clinically to assess postural stability (25). Table 3 summarises the results of the stepwise regression. In the group with PD, total body lean mass, leg fat mass, sex, age, and disease stage significantly predicted postural instability.

\section{Discussion}

We found a significant difference in postural stability between those with PD and an age- and sex-matched control group as measured by the SOT equilibrium composite score, the MCT, the TUG Tests and the Step Test. The SOT strategy composite score and the MCT latency composite scores were not significantly different. There was a significant difference between groups for both the TUG B $(p<.001)$ and the high cognition TUG H $(\mathrm{p}<.001)$ tests.

We established a tenuous association between postural stability and two body composition variables (total lean body mass and leg fat mass); postural stability was similarly associated with sex, age and disease status. Of the body composition variables, total body lean mass, appendicular lean mass and leg fat mass most strongly predicted variations in postural stability. For the posturography measures of postural stability, only the latency composite score showed a relationship with body composition variables, which was a positive association with the total body lean mass variable.

Female sex was a negative predictor for the equilibrium composite score. This agrees with previous studies showing female gender to be a significant 
Table 2

Results for postural stability tests and body composition

\begin{tabular}{|c|c|c|c|c|c|c|}
\hline \multirow[t]{2}{*}{ Tests } & \multirow[t]{2}{*}{ Test variables } & \multirow[t]{2}{*}{ Groups } & \multirow[t]{2}{*}{ Mean } & \multicolumn{2}{|c|}{$95 \%$ confidence interval } & \multirow[t]{2}{*}{ t-test ( $p$ value) } \\
\hline & & & & Lower bound & Upper bound & \\
\hline \multirow[t]{4}{*}{ Sensory organisation test } & Equilibrium & PD & 64.2 & 60.1 & 68.3 & $-2.03(\mathrm{p}<.04)$ \\
\hline & composite score & ${ }^{*}$ Con & 69.5 & 66.2 & 72.8 & \\
\hline & Strategy $(\%)$ & IPD & 79.1 & 77.0 & 81.1 & $* *-0.13$ \\
\hline & composite score & Con & 79.3 & 76.4 & 82.2 & \\
\hline \multirow[t]{2}{*}{ Motor control test } & Latency ( $(\mathrm{ms})$ composite score & PD & 131.3 & 127.7 & 135.0 & ** -0.74 \\
\hline & & Con & 133.1 & 129.9 & 136.2 & \\
\hline \multirow[t]{8}{*}{ Clinical tests } & | | TUG B (キs) & PD & 9.8 & 9.0 & 10.7 & $3.44(\mathrm{p}<.001)$ \\
\hline & & Con & 8.1 & 7.6 & 8.6 & \\
\hline & ITUG H (‡s) & PD & 10.9 & 9.8 & 12.0 & $4.52(\mathrm{p}<.001)$ \\
\hline & & Con & 8.1 & 7.6 & 8.7 & \\
\hline & $\S$ Step test (right)* & PD & 13.9 & 13.0 & 14.7 & $-3.64(\mathrm{p}<.001)$ \\
\hline & & Con & 15.7 & 15.1 & 16.3 & \\
\hline & $\S$ Step test (left)* & IPD & 13.5 & 12.5 & 14.5 & $-4.76(\mathrm{p}<.001)$ \\
\hline & & Control & 16.1 & 15.5 & 16.7 & \\
\hline \multirow[t]{12}{*}{ Body composition } & Total body lean mass (kg) & IPD & 49.1 & 46.4 & 51.9 & **1.666 \\
\hline & & Control & 46.1 & 43.7 & 48.5 & \\
\hline & Total leg lean mass $(\mathrm{kg})$ & IPD & 16.8 & 15.7 & 17.8 & **1.541 \\
\hline & & Control & 15.7 & 14.8 & 16.6 & \\
\hline & Appendicular lean mass (kg) & IPD & 22.3 & 20.8 & 23.7 & **1.636 \\
\hline & & Control & 20.7 & 19.4 & 22.0 & \\
\hline & Total body fat mass (kg) & IPD & 23.4 & 20.1 & 26.7 & **-0.332 \\
\hline & & Control & 24.1 & 21.5 & 26.7 & \\
\hline & Total leg fat mass (kg) & IPD & 8.0 & 6.8 & 9.3 & **-0.549 \\
\hline & & Control & 8.5 & 7.4 & 9.5 & \\
\hline & Percentage body fat $(\%)$ & IPD & 30.2 & 26.8 & 33.6 & **-1.288 \\
\hline & & Control & 33.0 & 30.3 & 35.7 & \\
\hline
\end{tabular}

*Control; **Not significant; tmillisecond; łseconds; §number of steps in 15 seconds; I I TUG B: Timed Up and Go Basic test; TUG H: 『Timed-Up and Go High cognition test

contributor to variance in balance control in $\operatorname{PD}(26,27)$. Age and disease were found to be positive predictive factors for postural stability as reported by previous studies (11). There were no significant differences in BMI, body composition variables or body composition phenotype, which is not consistent with previous studies. For example, in a meta-analysis of seven studies (28), people with $\mathrm{PD}$ had a significantly lower BMI than the comparison group, correlated with disease staging. Also, DXA results in the present study showed no participants with sarcopenic obesity, which is consistent with the findings of Toth et al (29), but inconsistent with other studies reporting the presence of sarcopenic obesity in those with PD (18-21).

Notably, only three of our participants with PD were sarcopenic. This is an unusual finding, as PD is often linked with sarcopenia (18-21). Obesity is not a body composition phenotype normally associated with PD, yet one-third of our participants with PD were found to be obese. Revilla et al showed total fat and percentage of fat were higher, and the total lean mass was lower, in males with PD when compared with male controls, suggesting a sarcopenic phenotype; females with PD and female controls had similar values (19). Thus, our findings with regard to obesity in individuals with PD are unique and may reflect rising levels of obesity in the general older adult population (15).

Potential study limitations include the cross sectional design, the fact that participants with PD were primarily in a mild to moderate staging of their disease, which may 
Table 3

Summary of the stepwise regression results

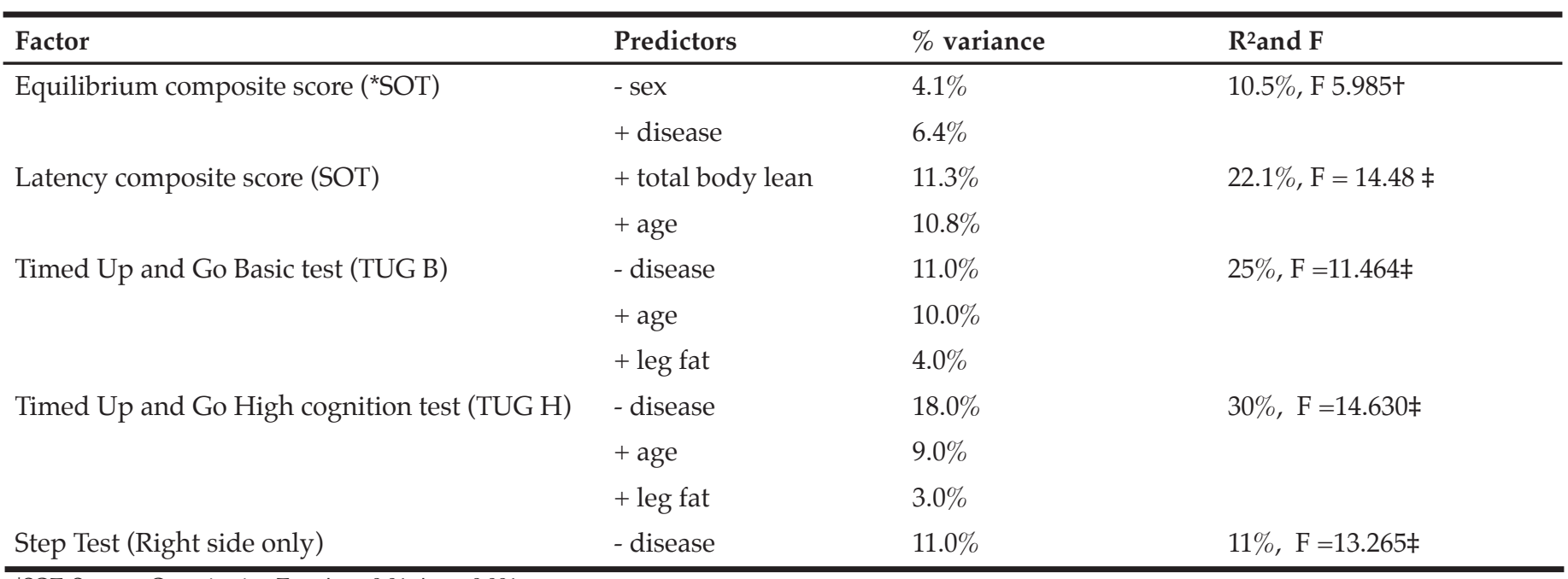

*SOT: Sensory Organization Test, $\mathrm{t}=\mathrm{p}<0.01, \neq=\mathrm{p}<0.001$

account for some differences in our results and those of previous studies, and the criteria used to define the body composition phenotypes. Although there are now several published operational criteria, in the current study we used criteria recommended by Baumgartner et al (15) and not the criteria set which includes muscle function/strength, as this is still widely debated in the literature. The main study strengths were the relatively large sample size, and case matching to a control group.

Although postural instability is a frequent manifestation of PD, the reasons are not fully understood and most likely a complex interplay of numerous factors is responsible. Our study investigated body composition as one such contributing factor, and a small association was identified. While the group with PD were significantly different with regard to measures of postural stability, body composition variables were not significantly different. Unexpected findings were that only three participants were found to be sarcopenic, and a large number of participants with PD were obese, in contrast with findings in the current literature. The findings of the current study could have significant implications for the clinical treatment of the physical symptoms of those suffering from PD.

Funding: This work was supported by a grant from The Physiotherapy New Zealand Scholarship Trust Fund. The first author received financial assistance from The Iraqi Ministry of Higher Education and Scientific Research/ Mission Office and the School of Physiotherapy, College of Health and Medical Technology, Baghdad, Iraq. The sponsors had no role in the design and conduct of the study; in the collection, analysis, and interpretation of data; in the preparation of the manuscript; or in the review or approval of the manuscript.

Conflict of interest: There are no conflicts of interest.

Ethical Standards: Ethical approval was granted by the Lower South Regional Ethics Committee, New Zealand (Ref: LRS/10/10/047).

\section{References}

1. Morgante L, Salemi G, Meneghini F, et al. Parkinson disease survival - A population-based study. Archives of Neurology 2000;57:507-512.

2. Ashburn A, Stack E, Pickering RM, Ward CD. A community-dwelling sample of people with Parkinson's disease: characteristics of fallers and non-fallers. Age Ageing 2001; 30:47-52.

3. Beuter A, Hernández R, Rigal R, Modolo J, Blanchet PJ. Postural sway and effect of levodopa in early Parkinson's disease. Can J Neurol Sci 2008;35:6568.

4. Bleuse S, Cassim F, Blatt J-L, et al. Anticipatory postural adjustments associated with arm movement in Parkinson's disease: a biomechanical analysis. J Neurol Neurosurg Psychiatry 2008;79:881-887.

5. Rossi M, Soto A, Santos S, Sesar A, Labella T. A prospective study of alterations in balance among patients with Parkinson's Disease. Protocol of the postural evaluation. Eur Neurol 2009;61:171-176.

6. Brown LA, Cooper SA, Doan J.B, et al. Parkinsonian deficits in sensory integration for postural control: temporal response to changes in visual input. Parkinsonism Relat Disord 2006;12) 376-381.

7. Bartolic A, Pirtosek Z, Rozman J, Ribaric S. Postural stability of Parkinson's disease patients is improved by decreasing rigidity. Eur J Neurol 2005;12:156159.

8. Morris, ME. Movement disorders in people with Parkinson disease: a model for physical therapy. Phys Ther 2005;80:578-597.

9. Paul SS, Sherrington C, Fung VS, Canning CG. Motor and cognitive impairments in Parkinson disease: relationships with specific balance and mobility tasks. Neurorehabil Neural Repair 2013;27:63-71.

10. Diab KS, Hale LA, Waters DL, Skinner MA. Factors contributing to postural instability in patients with idiopathic Parkinson's disease. Physical Therapy Reviews 2014;19:302-327.

11. Bosek M, Grzegorzewski B, Kowalczyk A, Lubinski I. Degradation of postural control system as a consequence of Parkinson's disease and ageing. Neurosci Lett 2005;376:215-220.

12. Ambrose AF, Paul G, Hausdorff JM. Risk factors for falls among older adults: a review of the literature. Maturitas 2013;75:51-61.

13. Waters DL, Hale LA, Grant AM, Herbison P, Goulding A. Osteoporosis and gait and balance disturbances in older sarcopenic obese New Zealanders. Osteoporos Int 2010;21:351-357.

14. Prado CM, Siervo M, Mire E, et al. A population-based approach to define body-composition phenotypes. Am J Clin Nutr 2014;99:1369-1377.

15. Baumgartner RN, Wayne SJ, Waters DL, Janssen I, Gallagher D, Morley JE. Sarcopenic obesity predicts instrumental activities of daily living disability in the elderly. Obes Res 2004;12:1995-2004.

16. Bouchard DR, Dionne IJ, Brochu M. Sarcopenic/obesity and physical capacity in older men and women: data from the Nutrition as a Determinant of Successful Aging (NuAge)-the Quebec longitudinal Study. Obesity (Silver Spring) 2009;17:2082-2088.

17. Poehlman ET, Toth MJ, Fishman PS, et al. Sarcopenia in aging humans: the impact of menopause and disease. J Gerontol A Biol Sci Med Sci 1995;50:7377.

18. Revilla M, Jiménez-Jiménez FJ, Villa LF, et al. Body composition in 
Parkinson's disease: a study with dual-energy X-ray absorptiometry. Parkinsonism Relat Disord 1998;4:137-142.

19. Petroni ML, Albani G, Bicchiega V, et al. Body composition in advancedstage Parkinson's disease. Acta Diabetol 2003;40:S187-190.

20. Doherty J, Farid S, Avalos G, Ryan M, Rooney B, Hickey P. Falls and disability in a study of sarcopenia and bone health in parkinson's disease. Osteoporos International 2011;22:S352-S353.

21. Lorefat B, Ganowiak W, Wissing U, Granerus AK, Unosson M. Food habits and intake of nutrients in elderly patients with Parkinson's disease. Gerontology 2006;52:160-168.

22. Hoehn MM, Yahr MD. Parkinsonism: onset, progression and mortality. Neurology 1967;17:427-442.

23. Hill KD, Bernhardt J, McGann AM, Maltese D, Berkovis D. A New Test of Dynamic Standing Balance for Stroke Patients: Reliability, Validity and Comparison with Healthy Elderly. Physiother Canada 1996;48:257-262.

24. Smithson F, Morris ME, Iansek R. Performance on clinical tests of balance in Parkinson's disease. Phys Ther 1998;78:577-592.
25. Toole T, Park S, Hirsch MA, Lehman DA, Maitland CG. The multicomponent nature of equilibrium in persons with parkinsonism: a regression approach. J Neural Transm 1996;103: 561-580.

26. Schenkman M, Morey M, Kuchibhatla M. Spinal flexibility and balance control among community-dwelling adults with and without Parkinson's disease. J Gerontol A Biol Sci Med Sci 2000;55:M441-445.

27. Roland KP, Jakobi JM, Powell C, Jones GR. Factors related to functional independence in females with Parkinson's disease: a systematic review. Maturitas 2011;69:304-311.

28. Morales-Briceno H, Cervantes-Arriaga A, Rodríguez-Violante M, CallejaCastill J, Corona T. Overweight is more prevalent in patients with Parkinson's disease. Arq Neuropsiquiatr 2012;70:843-846.

29. Toth MJ, Fishman PS, Poehlman ET. Free-living daily energy expenditure in patients with Parkinson's disease. Neurology 1997;48:88-91. 